OPEN ACCESS

Edited by: Davide Rassati,

University of Padova, Italy

Reviewed by:

Jonathan David Sweeney, Canadian Forest Service, Canada

Kevin J. Dodds,

United States Forest Service,

United States

*Correspondence:

Therese M. Poland

therese.poland@usda.gov

†Present address:

Tina M. Ciaramitaro,

Auburn University School of Forestry and Wildlife Sciences, Auburn, AL,

United States

Specialty section

This article was submitted to

Forest Disturbance,

a section of the journal

Frontiers in Forests and Global

Change

Received: 16 August 2019 Accepted: 14 November 2019 Published: 05 December 2019

Citation:

Poland TM, Petrice TR and Ciaramitaro TM (2019) Trap Designs,

Colors, and Lures for Emerald Ash Borer Detection.

Front. For. Glob. Change 2:80 doi: 10.3389/ffgc.2019.00080

\section{Trap Designs, Colors, and Lures for Emerald Ash Borer Detection}

\author{
Therese M. Poland ${ }^{*}$, Toby R. Petrice and Tina M. Ciaramitaro ${ }^{\dagger}$ \\ USDA Forest Service, Northern Research Station, Lansing, MI, United States
}

The emerald ash borer, Agrilus planipennis, is the most damaging invasive forest insect pest ever to have invaded North America. It is native to Asia and is established in the United States, Canada, European Russia and Ukraine where it threatens native ash across North America and Europe. We evaluated trap designs, colors, and lures for A. planipennis detection at sites with varying infestation levels. Purple or green sticky prism traps and multiple funnel traps hung in the canopy of ash trees and double-decker traps (consisting of two sticky prisms attached to a $3 \mathrm{~m}$ vertical pole at $3 \mathrm{~m}$ and $1.8 \mathrm{~m}$ above ground) had high detection rates even at sites with very low infestation levels. At a low infestation site, females were more attracted to dark purple sticky prism traps hung in the canopy and to Manuka oil and Phoebe oil lures than to light green sticky prism traps or cis-3-hexenol lures; whereas, males were more attracted to light green sticky prism traps in the canopy and cis-3-hexenol lures than to dark purple sticky prism traps or Manuka and Phoebe oil lures. More males and females were captured in double-decker traps with dark green upper prisms and light purple lower prisms, baited with cis-3-hexenol, than in double-decker traps with dark purple upper and lower prisms. Dark green funnel traps and double-decker traps with dark green upper and light purple lower prisms baited with cis-3-hexenol lures captured more females than dark green sticky prism traps hung in the canopy at sites with very low infestation levels. Detection rates were similar among trap types and ranged from 75 to $80 \%$ for dark green sticky prism traps, 82.5-100\% for dark green funnel traps, and 100\% for double-decker traps with dark green upper and light purple lower prisms at sites with very low $A$. planipennis infestations. Cost, ability to reuse the traps, and ease of deployment varies among trap types. These and other factors including trap placement, host density and condition should be considered in selecting traps and designing operational surveys. Future research is needed to determine effective trapping radius, relationship of trap catches to population density, cost benefit of different trap types, and optimal deployment strategy. Greater numbers of $A$. planipennis captured and higher detection rates in cis-3-hexenol-baited double-decker traps with dark green upper prisms and light purple lower prisms and in dark green funnel traps compared to dark green prism traps at sites with very low infestation levels, suggest these trap types would be most effective for operational detection surveys.

Keywords: Agrilus planipennis, invasive species, early detection, multiple funnel trap, prism trap, double-decker trap, color, cis-3-hexenol 


\section{INTRODUCTION}

The emerald ash borer, Agrilus planipennis Fairmaire (Coleoptera: Buprestidae), a wood-boring beetle native to Asia, is the most damaging invasive forest insect pest to have invaded North America (Herms and McCullough, 2014). It was likely introduced in the mid-1990s (Siegert et al., 2014), but was not discovered until 2002 near Detroit, Michigan (Haack et al., 2002; Poland and McCullough, 2006). This invasive pest is spreading rapidly and threatens to eliminate native ash (Fraxinus spp.) as an overstory component in North American forests (Herms and McCullough, 2014). It has also been detected and is spreading in European Russia and Ukraine (Haack et al., 2015; Orlova-Bienkowskaja et al., 2019) and is of great concern to other European countries (Orlova-Bienkowskaja, 2014; Musolin et al., 2017).

Early detection and monitoring are critically important for managing invasive species because management becomes increasingly difficult as populations expand and build to high densities (Brockerhoff et al., 2010). Discovering an invasive species at an early stage of invasion before it becomes widespread allows the rapid implementation of risk mitigation measures, efficient and environmentally sound management decisions, and responses to prevent or slow its spread (Liebhold and Tobin, 2008). Developing optimal detection strategies and tools increases the likelihood for early detection and successful invasive species management programs (Tobin et al., 2014). Effective traps are also essential for monitoring established populations to determine relative density of infestations, spread of invasions and location of hot spots, and to evaluate the effectiveness of management strategies and control tactics (Lyons et al., 2008).

Many factors influence trap efficacy including intrinsic trap characteristics such as color, design, and olfactory attractants, as well as extrinsic conditions such as vertical and horizontal trap placement, environmental surroundings (e.g., host abundance, distribution, and condition, and land use such as ports-of-entry, industrial areas, campgrounds, new developments, or forests and natural areas), and population density (Rassati et al., 2015; Poland and Rassati, 2018). There has been considerable research to develop effective traps and other detection tools including trap trees for early detection of A. planipennis (McCullough et al., 2009a,b; Ryall, 2015). Electroretinogram assays have demonstrated both male and female A. planipennis adults are sensitive to specific wavelengths of violet and green light whereas only females are sensitive to red wavelengths (Crook et al., 2009). Males also orient visually to mates and land on dead pinned females placed on the trunks or leaves of ash trees (Rodriguez-Saona et al., 2007; Lelito et al., 2008) or on artificial decoys (Domingue et al., 2015). In addition to visual cues, A. planipennis adults use long- and short-range olfactory cues to locate hosts and mates. Ash volatiles, including bark sesquiterpenes and green leaf volatiles, elicit antennal responses by A. planipennis adults (Rodriguez-Saona et al., 2006; Crook et al., 2008). In field studies, adults were attracted to traps baited with the leaf alcohol cis-3-hexenol found in the volatiles from ash leaves (Rodriguez-Saona et al., 2006; de Groot et al., 2008; Grant et al., 2010) and natural tree oils including
Manuka oil [from the New Zealand tea tree, Leptospermum scoparium J. R. and C. Forst (Myrtaceae)] and Phoebe oil [from the Brazilian walnut tree, Phoebe porosa Mez. (Lauraceae)] which contain sesquiterpenes found in ash bark volatiles (Crook et al., 2008; de Groot et al., 2008; Crook and Mastro, 2010; Grant et al., 2010, 2011; Poland et al., 2011). Females produce a combination of contact pheromones that change with reproductive maturity (Lelito et al., 2009; Silk et al., 2009) as well as a short-range volatile pheromone cis-3-lactone which has been shown to increase attraction to traps baited with cis-3-hexenol (Silk et al., 2011, 2019; Ryall et al., 2012; Ryall, 2015).

Several different trap designs have been developed and evaluated for capture of $A$. planipennis. Early trap designs included box-shaped traps and cross-vane panel traps of different colors (Francese et al., 2008). As our understanding of $A$. planipennis behavior and responses to colors and volatiles advanced (Lelito et al., 2007; Rodriguez-Saona et al., 2007; Crook et al., 2009, 2012; Francese et al., 2010a) trap colors were optimized to specific shades of green or purple and new trap designs were developed. Three-sided prism traps presented a wider silhouette than box or cross-vane traps for the same amount of material and the flat surface resulted in higher reflectance of colors compared to cross-vane traps and a broader sticky area for insect capture (Francese et al., 2008). Sticky cards or leaves with pinned dead A. planinpennis adults (Lelito et al., 2008; Domingue et al., 2013; Petrice et al., 2013) and branch traps with artificial beetle decoys (Domingue et al., 2015) have been tested to exploit the observation that male A. planipennis respond visually to females and land on them in the canopies of ash trees (Lelito et al., 2008). Green and/or purple doubledecker traps were designed to incorporate multiple attractive stimuli of ash trees. They are composed of a $10 \mathrm{~cm}$ diameter, $3 \mathrm{~m}$ tall PVC pole with an upper prism trap attached to the top and a lower prism attached $60 \mathrm{~cm}$ below which are baited separately with leaf and bark volatiles, respectively, to represent the tree canopy and bole (Poland et al., 2011). More recently, green or purple multiple-funnel traps with different slippery coatings that can be re-used for multiple years have been tested as an alternative to sticky traps that must be discarded (Francese et al., 2011, 2019; Crook et al., 2014).

In field trapping experiments, green $(\lambda=530-540 \mathrm{~nm}$ wavelengths) and purple (with peak wavelengths at $\lambda=430$, 600 , and $670 \mathrm{~nm}$ ) traps were found to be highly attractive to $A$. planipennis (Crook et al., 2009, 2012; Francese et al., 2010a,b, 2013a). Decreasing the reflectance of green traps from $67 \%$ to $49 \%$ resulted in a darker shade of green and significantly increased the number of adults captured (Francese et al., 2010a), while altering the shade of purple $(430 \mathrm{~nm} 20 \%$ reflectance,

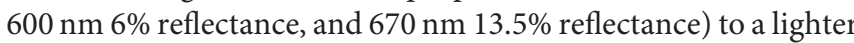

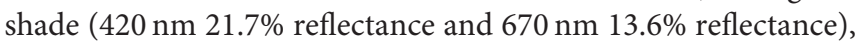
referred to as Sabic purple, also increased attraction (Francese et al., 2010a, 2011, 2013a). Purple traps were found to be more attractive to females than to males (Crook et al., 2009; Francese et al., 2010b, 2013a).

Trap placement influences A. planipennis captures, most likely due to male and female behaviors. In China and North America, A. planipennis adults are more active in the afternoon in sunny locations and prefer to attack trees grown in full 
sun rather than in the shade (Yu, 1992; McCullough et al., 2009a,b). Males tend to hover around the canopies of ash trees before landing on females to mate or on leaves to feed (Rodriguez-Saona et al., 2007; Lelito et al., 2008). Ryall et al. (2013) captured more A. planipennis when green sticky prism traps were placed in the south or sun-exposed aspect of tree canopies compared to shaded aspects. Trap height may also affect capture rates. For instance, Crook et al. (2008) found that more A. planipennis were captured on purple prism traps placed high in the canopy compared with similar traps placed below the canopy.

Tree condition and A. planipennis infestation levels can also influence trap captures. At high A. planipennis densities, heavily infested trees may emit stress-induced volatiles (RodriguezSaona et al., 2006) which may enhance attraction to traps hung in infested trees or compete with traps hung in nearby uninfested trees. As canopy dieback progresses in heavy infestations, light availability increases and hyperspectral signatures of trees are altered (Bartels et al., 2008), potentially affecting beetle response to trap color or placement. The number of $A$. planipennis captured in traps hung in the ash canopy was positively correlated with the infestation level of the tree in which they were hung (Poland and Mccullough, 2014).

The US Department of Agriculture, Animal and Plant Health Inspection Service, Plant Protection and Quarantine (USDA APHIS-PPQ) Emerald Ash Borer Cooperative Program conducts a multi-state emerald ash borer survey program that relies on traps. The program has evolved with advances in detection techniques from visual surveys in 2002, to girdled trap trees in 2005, and implementation of glue-coated dark purple prism traps baited with Manuka oil in 2008 (USDAAPHIS-PPQ, 2018). Based on positive detection results and feedback, the APHIS survey continues to rely primarily on purple prism traps which are currently baited with cis-3-hexnol lures, with 12,000 set out in 2018 in a buffer zone along the leading edge of the infestation. Dark green multiple funnel traps baited with cis-3-hexenol are also recommended as an alternative and used in some locations (USDA-APHIS-PPQ, 2018; Francese et al., 2019). In Canada, visual and branch sampling surveys along with green prism traps hung in the canopy of ash trees and baited with cis-3-hexenol and cis-3lactone are the recommended tools for A. planipennis detection surveys (CFIA, 2019; Silk et al., 2019). Regulatory agencies review and update survey protocols and guidelines annually based on recommendations from science advisory panels, logistical and economic considerations, and consistency of methods and messaging from year to year for monitoring, training, and public outreach purposes.

Although many studies have compared various trap types, colors, and lures (Marshall et al., 2009, 2010b; McCullough et al., 2011; Poland et al., 2011; Crook et al., 2012, 2014; Fierke et al., 2013; Francese et al., 2013a; Poland and Mccullough, 2014), results have been variable (Ryall, 2015). Several studies have focused on optimizing the color of sticky prism traps or multiple funnel traps (Crook et al., 2009, 2012; Francese et al., 2010a,b, 2013a), while other studies have compared sticky prism traps baited with different lures (Crook et al.,
2008; de Groot et al., 2008; Grant et al., 2010, 2011; Silk et al., 2011; Ryall et al., 2013; Ryall, 2015). Most studies have been conducted at sites with moderate to high $A$. planipennis infestation levels which are not representative of newly invaded areas with low infestation levels where detection surveys would occur (Burr et al., 2018). Few studies have compared multiple trap types of different colors and baited with different lures to determine optimal recommendations for operational detection programs. Also, optimal colors have not been previously tested for double-decker traps and they have not been compared to multiple trap types including prism traps, and multiple funnel traps.

Our objective was to evaluate the most promising trap designs, colors, and lures for A. planipennis detection in order to improve operational survey programs. Using field sites with varying levels of $A$. planipennis infestation, we compared multiple combinations of lures, colors, and trap types including sticky prism and multiple funnel traps hung in the ash canopy and double-decker traps at ground level.

\section{METHODS}

Four trapping experiments were conducted from 2010 to 2015 to compare efficacy of the most promising trap designs for capturing A. planipennis (Table 1). Experiments were conducted at field sites in southern Michigan differing in infestation levels of $A$. planipennis based on visible signs and symptoms such as woodpecker attacks, epicormic shoots, and percentage of crown dieback. In 2010, field sites included the MSU Kellogg Experimental Forest, Augusta, Kalamazoo County; Legg Park, Okemos, Ingham County; and Fox Memorial Park, Potterville, Eaton County. The site at the Kellogg Experimental Forest consisted of a small stand of green ash, Fraxinus pennsylvanica Marshall, trees with a moderate infestation of A. planipennis. Some trees had evidence of A. planipennis infestation including woodpecker feeding holes, epicormic shoots, and about 25$30 \%$ crown dieback. Legg Park consisted of a natural area along the Red Cedar River with open fields surrounded by a mixed hardwood forest with a major component of mature green ash. Fox Memorial Park consisted of an open area with a small lake surrounded by mixed hardwoods including a major component of green ash. At Legg Park and Fox Memorial Park, A. planipennis infestations were very high with many heavily infested trees having abundant woodpecker attacks, epicormic shoots and $>30 \%$ crown dieback. In 2014, field sites included Legg Park, Fox Memorial Park, and a private woodlot near Ithaca, Gratiot County, MI. Infestations at Legg Park and Fox Memorial Park had passed their peak and A. planipennis populations had declined to very low levels, with few surviving mature ash trees and many small healthy saplings. The private woodlot near Ithaca included a major component of green ash, many of which had symptoms of infestation including woodpecker attacks, epicormic shoots, and $35-40 \%$ crown dieback. The population of A. planipennis was high and the infestation level was nearing its peak. In 2015, the experiment was conducted at Legg Park where 
TABLE 1 | Field sites and treatments for A. planipennis trapping experiments in southern Michigan, 2010-2015.

\begin{tabular}{|c|c|c|c|c|}
\hline Experiment number (Year) & Field site & A.planipennis infestation level & Number of blocks & Treatments \\
\hline \multirow[t]{4}{*}{1 (2010) } & Kellogg Forest & Moderate & 2 & 1) Dark purple and light green prisms unbaited Control \\
\hline & Legg Park & High & 8 & 2) Dark purple and light green prisms Hex \\
\hline & Fox Memorial & Very high & 7 & 3) Dark purple and light green prisms Man/Phoebe \\
\hline & & & & 4) Dark purple and light green prisms Hex + Man/Phoebe \\
\hline \multirow[t]{5}{*}{$2(2014)$} & Legg Park & Very low & 10 & 1) Dark purple DD HexHex \\
\hline & & & & 2) Dark purple DD HexMan \\
\hline & & & & 3) Light purple DD HexMan \\
\hline & & & & 4) Green/purple DD HexHex \\
\hline & & & & 5) Green/purple DD HexMan \\
\hline \multirow[t]{4}{*}{$3(2014)$} & Fox Memorial & Very low & 5 & 1) Dark green funnel Hex \\
\hline & Ithaca & High & 5 & 2) Dark green prism Hex \\
\hline & & & & 3) Green/purple DD HexHex \\
\hline & & & & 4) Dark purple DD HexMan \\
\hline \multirow[t]{3}{*}{$4(2015)$} & Legg Park & Very low & 8 & 1) Dark green funnel Hex \\
\hline & & & & 2) Dark green prism Hex \\
\hline & & & & 3) Green/purple DD HexHex \\
\hline
\end{tabular}

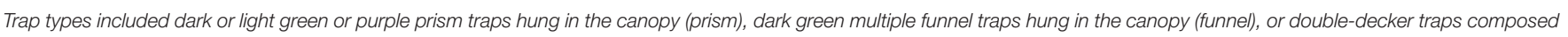

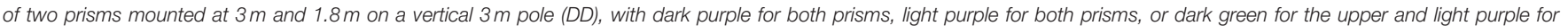

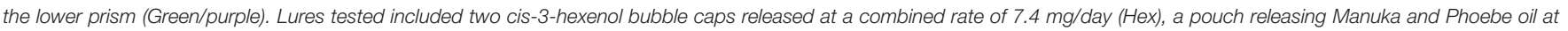

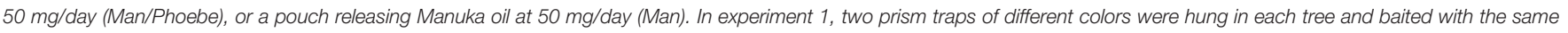
lure treatment.

the A. planipennis infestation had collapsed and populations remained very low.

We tested three different trap types in various colors. Sticky prism traps consisted of 3-sided corrugated plastic prisms (60 $\times 40 \mathrm{~cm}$ on each side) coated with Pestick insect trapping glue (Hummert International, Earth City, MO). They were hung in the upper half of the canopies of ash trees along the edge of a stand approximately 6-10 $\mathrm{m}$ above ground. Traps were hung from rope installed over the end of a branch extending into the opening at the edge of the stand using a Big Shot Line Launcher (Sherrill Tree, Greensboro, NC). Double-decker traps consisted of a 3-m tall PVC pipe $(10 \mathrm{~cm}$ diameter) set over a T-post in the ground. Two sticky prism traps coated with Pestick were attached to the PVC pipe: an upper prism at the top and a lower prism $60 \mathrm{~cm}$ below the upper prism (Poland et al., 2011). Double-decker traps were placed about 5-10 m from the edge of a stand. Multiple funnel traps were standard 12-unit traps (Synergy Semiochemical, Delta, B.C.) and were coated with 10\% Fluon, a slippery fluoropolymer (AGC Chemicals Americas, Inc., Exton, PA) which has been shown to enhance captures of woodboring beetles (Graham and Poland, 2012; Allison, 2016). We tested four colors: (1) dark purple $(\lambda=430 \mathrm{~nm} 20 \%$ reflectance, $600 \mathrm{~nm}$ $6 \%$ reflectance, and $670 \mathrm{~nm} 13.5 \%$ reflectance, Harbor Sales Inc., Suddlersville, MD); (2) light purple ( $\lambda=420 \mathrm{~nm} 21.7 \%$

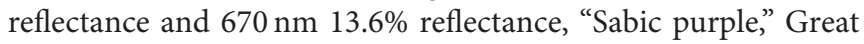
Lakes Integrated Pest Management, Vestaburg, MI); (3) light green $(\lambda=540 \mathrm{~nm}$, peak reflectance $64 \%$, Great Lakes Integrated Pest Management, Vestaburg, MI); and dark green $(\lambda=540 \mathrm{~nm}$, peak reflectance 49\%, Great Lakes Integrated Pest Management, Vestaburg, MI). The light shade of purple and dark shade of green were found to be more attractive to A. planipennis than the original dark purple and light green (Francese et al., 2010a, 2011, 2013a). Prisms of double-decker traps were either both the same color (e.g., "dark purple", or "light purple") or different colors for the upper and lower prisms (e.g., dark green upper and light purple lower, hereinafter "green/purple").

Traps were baited with either two cis-3-hexenol bubble caps (hereinafter "Hex") with a combined release rate of $7.4 \mathrm{mg} /$ day (previously available from the former Contech Enterprises Inc., Delta, B.C., currently similar lures available from Scentry Biologicals, Inc., Billings, MT; Synergy Semiochemicals, Delta, B.C.; Sylvar Technologies, Inc., Fredericton, NB), one pouch with an 80:20 blend of Manuka and Phoebe oil (hereinafter "Man/Phoebe") released at $50 \mathrm{mg} /$ day (Synergy Semiochemicals Inc., Delta, B.C.), one Manuka oil pouch (hereinafter "Man") released at $50 \mathrm{mg} /$ day (Synergy Semiochemicals Inc., Delta, B.C.) or different combinations. The upper and lower prisms of double-decker traps were baited separately to provide a point source of attraction for each trapping surface area, with either Hex lures on both prisms, or a Hex lure on the upper panel representing leaf volatiles in the canopy and a Man lure on the lower panel representing bark volatiles from the bole of trees.

Experiment 1 was conducted 12 May-4 August 2010 and compared dark purple and light green sticky prism traps. The experiment used a multi-location randomized complete block factorial design with 4 lure and 2 color treatments and 17 blocks. Two blocks were set up at the Kellogg Experimental Forest with a moderate $A$. planipennis infestation, 8 blocks at Legg Park with a high infestation, and 7 blocks at Fox Memorial Park with a very high infestation. Live ash trees were selected in which to hang the traps. Trees within a block were separated by at least $10 \mathrm{~m}$ and blocks were separated by at least $20 \mathrm{~m}$. In each tree, 


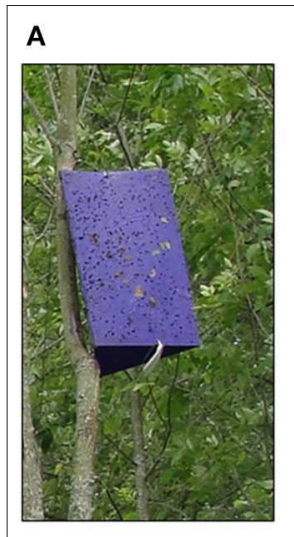

D

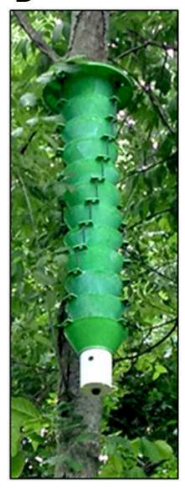

E

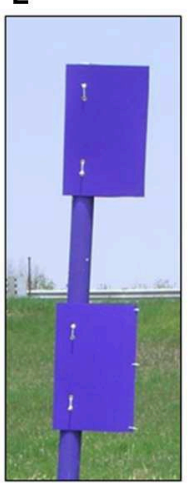

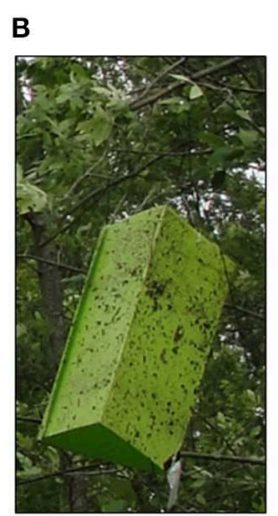

$\mathbf{F}$

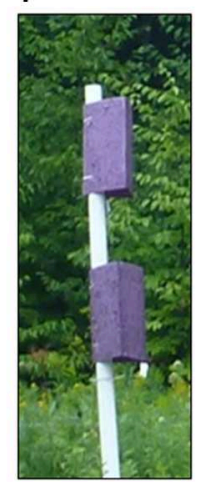

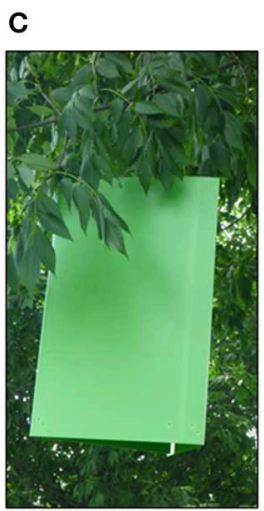

G

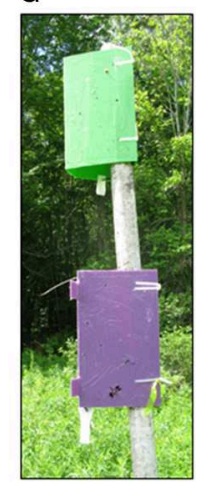

FIGURE 1 | Trap types used in Agrilus planipennis trapping experiments. (A) Dark purple sticky prism trap. (B) Light green sticky prism trap. (C) Dark green sticky prism trap. (D) Dark green funnel trap. (E) Double-decker trap with dark purple upper and lower prisms. (F) Double-decker trap with light purple upper and lower prisms. (G) Double-decker trap with dark green upper prism and light purple lower prism.

one dark purple (Figure 1A) and one light green (Figure 1B) sticky prism trap were randomly assigned to separate branches at least $3 \mathrm{~m}$ apart. Both traps in the same tree were baited with the same lure treatment. Each block consisted of four trees, each containing a purple and a green trap baited with one of four randomly assigned lure treatments: (1) Unbaited control; (2) Hex; (3) Man/Phoebe; or (4) Both Hex and Man/Pheobe. Lures were replaced on 12 to 13 July at which time infestation level of each tree containing traps was estimated. Infestation level was ranked as " 1 " if the tree was lightly infested with few visible symptoms and $<10 \%$ dieback, " 2 " if the tree was moderately infested with a few woodpecker feeding holes and epicormic shoots and $<35 \%$ dieback, and " 3 " if the tree was heavily infested and had abundant woodpecker feeding holes, epicormic shoots, and $>35 \%$ dieback.

Experiment 2 was conducted 12 June-11 August 2014 at Legg Park with a very low $A$. planipennis population after the first wave of infestation had declined. The experiment compared doubledecker traps with five different color and lure combinations including the standard dark purple used for the APHIS survey program and previous studies with double-decker traps (Poland et al., 2011) as well as newer shades of dark green and light purple that were found to enhance attraction of A. planipennis (Francese et al., 2010a, 2013a). There were 10 blocks of 5 treatments laid out in a randomized complete block design with at least $10 \mathrm{~m}$ between traps and $25 \mathrm{~m}$ between blocks: (1) Dark purple baited with Hex on both prisms (Dark Purple HexHex; Figure 1E); (2) Dark purple baited with Hex on the upper and Man on the lower prism (Dark Purple HexMan); (3) Light purple baited with Hex on both prisms (Light Purple HexHex; Figure 1F); (4) Green/purple baited with Hex on both prisms (Green/Purple HexHex; Figure 1G); and 5) Green/purple baited with Hex on the upper and Man on the lower prism (Green/Purple HexMan).

Experiment 3 was conducted 12 June-3 September 2014 and compared different trap types at two different sites, a private woodlot near Ithaca, MI with a high A. planipennis infestation level, and Fox Memorial Park with a very low population level after the initial outbreak had declined. The experiment had 10 blocks of 4 treatments, with 5 blocks at each site which were analyzed separately. The trap designs and colors tested included the most attractive double-decker trap from Experiment 2, the original dark purple double-decker trap design, and the funnel and prism traps in the newer dark green that was reported to enhance attraction of A. planipennis (Crook et al., 2012; Francese et al., 2013a,b). Treatments included: (1) Dark green multiple funnel trap baited with Hex (Green Funnel; Figure 1D); (2) Dark green sticky prism trap baited with Hex (Green Prism; Figure 1C); (3) Green/purple double-decker trap baited with Hex on both prisms (Green/purple HexHex); and (4) Dark purple double-decker trap baited with Hex on the upper and Man on the lower prism (Dark purple HexMan).

Experiment 4 was conducted 9 June-30 July 2015 and compared different trap types at Legg Park with a very low A. planipennis population level after the initial infestation had declined. The trap types compared were similar to Experiment 3 except that the dark purple double-decker trap which captured fewer A. planipennis than the Green/Purple double-decker trap was not included. The experiment included 8 blocks of 3 treatments: (1) Green funnel baited with Hex (Green Funnel); (2) Dark green sticky prism baited with Hex (Green Prism); and (3) Green/purple double-decker trap baited with Hex on both prisms (Green/Purple HexHex).

For all experiments, captured A. planipennis were collected every 2 weeks and soaked in Histoclear II (National Diagnostics, Atlanta, GA) to remove Pestick, except for those captured in multiple funnel traps. All A. planipennis were sexed and the total number captured of each sex for the entire season was summed for each trap. All analyses were conducted using the SAS 9.4 for Windows statistical package (SAS Institute, 2012) with an $\alpha$ level of 0.05 . For each experiment, data for the number of A. planipennis captured were analyzed by a generalized linear mixed model (PROC GLIMMIX) with separate models analyzed for males, females, and totals of both sexes. For experiment 1, color, treatment, and the interaction of color within treatment were tested as fixed effects. The level of infestation of the tree in which traps were hung and interaction between infestation level and treatment were tested when running through the steps of ANCOVA. Since the interaction term was not significant, only infestation level was included as a covariate in the model. 
Site, block within site, and the interaction between color and treatment within site were included as random effects in the model. The Kenward Rogers denominator degrees of freedom adjustment method was used as an option in all models. Degrees of freedom were rounded to the nearest whole number for ease of reporting in the results section. The response distribution was lognormal with the identity link function. Differences among treatments and colors were tested with the Tukey-Kramer means comparison procedure. Separate models were run for each site with color, treatment, and color within treatment as fixed effects, block as a random effect, and infestation level of the tree in which traps were hung as a covariate. For experiments 2, 3, and 4 , treatment was tested as a fixed effect and block as a random effect. The response distribution was gamma with the log link function. In all models the distribution and link function that had the best residuals in terms of meeting normality via the Shapiro-Wilks test and homogeneity of variance via the Levene's test were retained. For all experiments, the detection rates for each treatment, or percentage of traps that captured at least one beetle were reported.

\section{RESULTS}

In experiment 1 , we captured $14,080 \mathrm{~A}$. planipennis adults on prism traps hung in the canopy. Both trap color and lure treatment, but not their interaction, had significant effects on the number of $A$. planipennis captured, but responses varied for males and females, with females generally being more attracted to dark purple traps baited with Manuka and Phoebe oil and males to light green traps baited with cis-3-hexenol (Table 2). For all sites combined, significantly more females were captured in dark purple sticky prism traps than in light green sticky prism traps hung in the canopy $(F=3.81 ; P=0.05 ; d f=1,22)$ and in traps baited with Manuka and Phoebe oil alone or combined with cis3 -hexenol than in unbaited control traps $(F=3.03 ; P=0.03$; Table 2; $d f=3,10)$. The number of females captured in traps baited with cis-3-hexenol alone was intermediate (Table 2). In contrast, significantly more males were captured in traps baited with cis-3-hexenol alone or combined with Manuka and Phoebe oil, than in unbaited control traps $(F=7.67 ; P=0.005$; Table 2; $d f=3,10)$. The number of males captured in traps baited with Manuka and Phoebe oil alone was intermediate (Table 2). Detection rates (percentage of traps that captured at least one $A$. planipennis) were similar for sticky prism traps of either color and all lure treatments, with detection rates ranging from $97 \%$ to $100 \%$. The covariate, infestation level of the tree in which traps were hung, had a significant effect on the number of $A$. planipennis captured for both sexes, but there was no significant interaction with treatment. Significantly more A. planipennis were captured in traps hung in trees with heavy infestation levels $(134.9 \pm 18)$ than in trees with moderate $(93.8 \pm 10)$ or light infestation levels $(57.9 \pm 13)(F=9.64 ; P=0.003 ; d f=1,67)$.

The pattern of responses for males and females to different treatments and colors were similar at all sites; however, differences in responses to different treatments and colors tended to be more pronounced at the site with the lowest population density (Table 2). We captured an average $( \pm S E)$ of $66.3 \pm 16$ beetles per trap at the Kellogg Experimental Forest site that had a moderate A. planipennis population, $76.4 \pm 9$ beetles per trap at Legg Park with a high population, and $145.1 \pm 16$ per trap at Fox Memorial Park with a very high population. At the Kellogg Experimental Forest with the lowest population density, significantly more females were captured in traps baited with Manuka and Phoebe oil $(31.25 \pm 2.5)$ than in traps baited with cis-3-hexenol $(9.0 \pm 2.4)$, unbaited control traps $(9.5 \pm 1.0)$ or both lures $(13.3 \pm 2.9)$ combined $(F=12.3$; $d f=3,11 ; P=$ $0.008)$ and in dark purple $(18.1 \pm 2.8)$ than in light green (13.4 $\pm 2.4)$ sticky prism traps hung in the canopy $(F=4.6 ; d f=1$, $11 ; P=0.05)$. More males were captured in traps baited with cis-3-hexenol alone $(78.8 \pm 36.0)$ or combined with Manuka and Phoebe oil $(82.0 \pm 51.4)$ than in unbaited control traps $(14.5 \pm$ 6.1) or traps baited with Manuka and Phoebe oil alone $(27.3 \pm$ $4.2 ; F=4.17 ; d f=3,11 ; P=0.03)$ and in light green $(77.1$ $\pm 29.5)$ than in dark purple $(24.1 \pm 6.5)$ sticky prism traps hung in the canopy $(F=6.12$; df $=1,11 ; P=0.03)$. Because significantly more males were captured than females, overall significantly more $A$. planipennis of both sexes combined were captured in light green $(95.3 \pm 29)$ than in dark purple $(37.5 \pm$ 6.5) sticky prism traps hung in the canopy $(F=5.89 ; P=0.03$; $d f=1,11)$.

We captured 494 A. planipennis adults in experiment 2 at Legg Park, a site with very low infestation levels in 2014 (Table 3). The color of sticky prisms on the double-decker traps significantly affected catches but lure treatment did not (Table 3 ). When traps were baited with Hex, the green/purple doubledecker traps captured significantly more females, males, and total A. planipennis than did dark purple double-decker traps, while light purple double-decker traps had intermediate catches (Table 3). Similarly, when traps were baited with Hex on the upper and Man on the lower prism, significantly more males and total $A$. planipennis were captured on green/purple than on dark purple double-decker traps, whereas, female trap catch did not differ among trap color combinations. Within the same trap color combination, lure treatment had no effect on $A$. planipennis catches. The detection rate of green/purple doubledecker traps was substantially higher (100\%) than detection rates of dark purple double-decker traps (60\%, 70\%), regardless of lure.

We captured 1314 A. planipennis adults in experiment 3 with 213 at Fox Memorial Park and 1101 at the private woodlot near Ithaca. More A. planipennis were captured per trap near Ithaca $(55.1 \pm 11)$ with a high infestation level than at Fox Memorial Park $(10.1 \pm 2)$ where the A. planipennis infestation was past its peak and populations had declined to very low levels $(F=$ $31.5 ; d f=1,8 ; P=0.0006)$. At the site near Ithaca with a high A. planipennis population, significantly more males and females were captured in dark green funnel and dark green sticky prism traps hung in the canopy than in dark purple double-decker traps. The number of males and females captured in green/purple double-decker traps was intermediate (Table 4). All traps had $100 \%$ detection rates. At Fox Memorial Park with a very low infestation level, significantly more females were captured in dark green funnel traps and in green/purple double-decker than in 
TABLE 2 | Mean number ( \pm SE) of A. planipennis captured and detection rate (percentage of traps that captured at least one beetle) in Experiment 1 that compared green or purple sticky prism traps baited with a blend of Manuka and Phoebe oil (50 mg/day), cis-3-hexenol (7.4 mg/day), or both.

\begin{tabular}{|c|c|c|c|c|c|}
\hline \multirow[b]{2}{*}{ Trap color and lure } & \multirow[b]{2}{*}{ Number of traps } & \multicolumn{3}{|c|}{ Mean number of EAB captured $\pm \mathrm{SE}$} & \multirow[b]{2}{*}{ Detection rate $(\%)$} \\
\hline & & Females & Males & Total & \\
\hline \multicolumn{6}{|l|}{ Both colors } \\
\hline Control & 34 & $32.2 \pm 5.8 b$ & $31.3 \pm 5.0 b$ & $64.4 \pm 10.9 b$ & $97 \pm 3$ \\
\hline Manuka/Phoebe Oil & 34 & $46.8 \pm 6.6 \mathrm{a}$ & $42.86 .9 \mathrm{ab}$ & $94.8 \pm 15.1 \mathrm{ab}$ & $97 \pm 3$ \\
\hline cis-3-Hexenol & 34 & $38.5 \pm 5.3 \mathrm{ab}$ & $72.0 \pm 9.6 a$ & $128.2 \pm 21.1 \mathrm{a}$ & $100 \pm 0$ \\
\hline Both & 34 & $42.9 \pm 5.3 a$ & $76.4 \pm 12.2 \mathrm{a}$ & $126.6 \pm 18.9 a$ & $100 \pm 0$ \\
\hline \multicolumn{6}{|l|}{ All lures } \\
\hline Light Green & 68 & $26.6 \pm 2.8 b$ & $59.7 \pm 7.5 \mathrm{a}$ & $95.8 \pm 13.1 \mathrm{a}$ & $97 \pm 2$ \\
\hline Dark Purple & 68 & $53.7 \pm 4.6 \mathrm{a}$ & $51.6 \pm 5.6 a$ & $111.3 \pm 11.4 \mathrm{a}$ & $100 \pm 0$ \\
\hline \multicolumn{6}{|l|}{$F ;$ df; $P$} \\
\hline Color & & $3.81 ; 1,22 ; 0.05$ & $0.49 ; 1,22 ; 0.4$ & $3.41 ; 1,22 ; 0.08$ & \\
\hline Lure & & $3.03 ; 3,10 ; 0.03$ & $7.67 ; 3,10 ; 0.005$ & $4.26 ; 3,10 ; 0.03$ & \\
\hline Color $\times$ lure (site) & & $1.71 ; 3,10 ; 0.07$ & $1.12 ; 3,10 ; 0.4$ & $1.2 ; 3,10 ; 0.3$ & \\
\hline Infestation level of tree & & $17.57 ; 1,68 ;<0.0001$ & $5.83 ; 1,68 ; 0.02$ & $9.64 ; 1,68 ; 0.003$ & \\
\hline
\end{tabular}

Traps were hung in the canopies of ash trees along the edge of forest stands at three sites in Southern Michigan with varying emerald ash borer infestation levels in 2010 . Mean numbers captured within a column followed by different letters are significantly different, Tukey test, $P<0.05$.

TABLE 3 | Mean number ( \pm SE) of $A$. planipennis captured and detection rate (percentage of traps that captured at least one beetle) in Experiment 2 that compared different colored double-decker (DD) traps along the edge of a forest stand at Legg Park, Ingham County Michigan with a very low emerald ash borer population level in 2014.

\begin{tabular}{|c|c|c|c|c|}
\hline Trap color and treatment & \multicolumn{3}{|c|}{ Mean number of EAB captured $\pm \mathrm{SE}$} & Detection rate $(\%)$ \\
\hline Dark Purple DD HexMan & $0.8 \pm 0.3 b$ & $1.0 \pm 0.5 c$ & $1.8 \pm 0.8 b$ & $60.0 \pm 16$ \\
\hline Light Purple DD HexHex & $1.9 \pm 0.5 \mathrm{ab}$ & $3.8 \pm 1.5 \mathrm{ab}$ & $5.6 \pm 1.9 a$ & $90.0 \pm 10$ \\
\hline Green/Purple DD HexHex & $3.1 \pm 0.7 \mathrm{a}$ & $5.9 \pm 0.9 a$ & $8.9 \pm 1.5 \mathrm{a}$ & $100.0 \pm 0$ \\
\hline$F ; \mathrm{df} ; P$ & $4.17 ; 4,36 ; 0.007$ & $9.25 ; 4,36 ;<0.0001$ & $10.47 ; 4,36 ;<0.0001$ & \\
\hline
\end{tabular}

Double-decker traps consisted of a 3-m PVC pole with an upper prism trap mounted to the top and lower prism trap mounted $60 \mathrm{~cm}$ below. Double-decker traps included dark purple for both the upper and lower prisms (Dark Purple), light purple for both the upper and lower prisms (Light Purple), or green for the upper prism and light purple for the lower prism (Green/Purple). Traps were baited with cis-3-hexenol lures (7.4 mg/day) on both panels (HexHex) or cis-3-hexenol on the upper panel and Manuka oil (50 mg/day) on the lower panel (HexMan). Mean numbers captured within a column followed by different letters are significantly different, Tukey test, $P<0.05 . N=10$.

dark green sticky prism traps. Dark purple double-decker traps captured an intermediate number of females. Differences among treatments followed a similar pattern but were not significant for males. The detection rate was $100 \%$ for all traps except for the dark green sticky prism traps of which $80 \%$ captured at least one A. planipennis (Table 4).

The results for experiment 4 in 2015 at Legg Park were similar to those for experiment 3 and A. planipennis populations were similarly very low after the peak infestation had declined. We captured 379 A. planipennis adults in the experiment. Differences among treatments were only significant for females and dark green prism traps hung in the canopy captured fewer females than dark green funnel or green/purple double decker traps. The detection rates were $100 \%$ for double-decker traps, $87.5 \%$ for dark green funnel traps, and $75 \%$ for dark green prism traps (Table 5).

\section{DISCUSSION}

Our results indicate that prism traps and multiple funnel traps hung in the canopy of ash trees and double-decker traps were all effective at capturing A. planipennis and a high percentage of traps detected $A$. planipennis even at sites with very low infestation levels. However, mean catch of males and females varied among trap types, colors, and lures tested. At sites with high population levels, differences among treatments were less pronounced. In experiment 1 at the site with lowest population density, females were more attracted to purple prism traps hung in the canopy and to Manuka oil and Phoebe oil lures than to green prism traps hung in the canopy or cis-3-hexenol lures; whereas, males were significantly more attracted to green sticky prism traps in the canopy and cis3-hexenol lures than to purple sticky prism traps or Manuka 


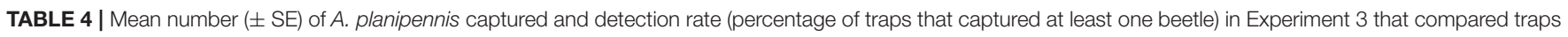

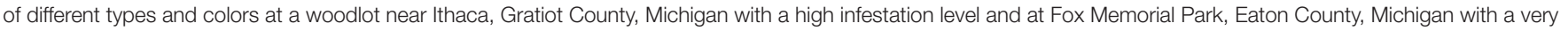
low infestation level in 2014.

\begin{tabular}{|c|c|c|c|c|}
\hline \multirow[b]{2}{*}{ Trap color and treatment } & \multicolumn{3}{|c|}{ Mean number of EAB captured \pm SE } & \multirow[b]{2}{*}{ Detection rate $(\%$} \\
\hline & Females & Males & Total & \\
\hline \multicolumn{5}{|l|}{ ITHACA } \\
\hline Green Funnel Hex & $39.8 \pm 15.1 \mathrm{a}$ & $41.4 \pm 16.6 \mathrm{a}$ & $81.2 \pm 31.3 a$ & $100 \pm 0$ \\
\hline Green Sticky Prism Hex & $36.4 \pm 8.7 \mathrm{a}$ & $44.6 \pm 17.6 \mathrm{a}$ & $81.0 \pm 22.6 . \mathrm{a}$ & $100 \pm 0$ \\
\hline Green/Purple DD HexHex & $12.8 \pm 1.4 \mathrm{ab}$ & $27.4 \pm 6.5 \mathrm{ab}$ & $40.2 \pm 7.1 \mathrm{ab}$ & $100 \pm 0$ \\
\hline Dark Purple DD HexMan & $10.2 \pm 3.3 b$ & $7.6 \pm 2.5 b$ & $17.8 \pm 5.4 b$ & $100 \pm 0$ \\
\hline$F ; \mathrm{df} ; P$ & $6.26 ; 3,16 ; 0.005$ & $56 ; 3,16 ; 0.008$ & $5.95 ; 3,16 ; 0.006$ & \\
\hline \multicolumn{5}{|l|}{ Fox Memorial Park } \\
\hline Green Funnel Hex & $8.4 \pm 3.4 \mathrm{a}$ & $7.6 \pm 2.9$ & $16.0 \pm 6.0 \mathrm{a}$ & $100 \pm 0$ \\
\hline Green Sticky Prism Hex & $1.6 \pm 1.1 b$ & $2.2 \pm 1.0$ & $3.8 \pm 2.2 b$ & $80 \pm 20$ \\
\hline Green/PurpleDD HexHex & $5.3 \pm 1.5 a$ & $6.8 \pm 3.1$ & $12.2 \pm 4.0 \mathrm{a}$ & $100 \pm 0$ \\
\hline Dark Purple DD HexMan & $4.0 \pm 1.4 \mathrm{ab}$ & $4.2 \pm 1.9$ & $8.2 \pm 3.2 \mathrm{a}$ & $100 \pm 0$ \\
\hline$F ; \mathrm{df} ; P$ & $4.78 ; 3,12 ; 0.02$ & $1.76 ; 3,12 ; 0.2$ & $3.48 ; 3,12 ; 0.04$ & \\
\hline
\end{tabular}

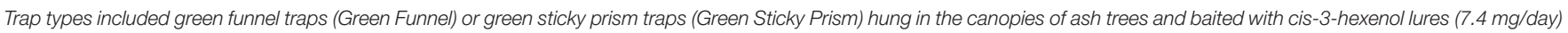

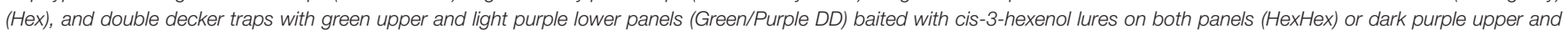

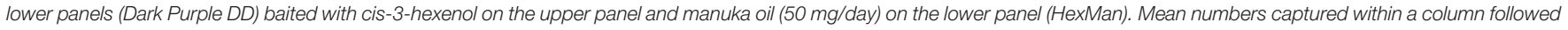
by different letters are significantly different, Tukey test, $P<0.05 . N=5$ blocks per site.

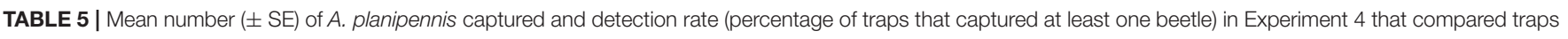
of different types and colors at a Legg Park, Ingham County, Michigan with a very low emerald ash borer infestation level in 2015.

\begin{tabular}{|c|c|c|c|c|}
\hline \multirow[b]{2}{*}{ Trap color and treatment } & \multicolumn{3}{|c|}{ Mean number of EAB captured $\pm \mathrm{SE}$} & \multirow[b]{2}{*}{ Detection rate (\%) } \\
\hline & Females & Males & Total & \\
\hline Green Funnel Hex & $4.8 \pm 1.9 \mathrm{a}$ & $7.8 \pm 3.0$ & $12.5 \pm 4.6$ & $87.5 \pm 12.5$ \\
\hline Green Sticky Prism Hex & $1.3 \pm 0.6 b$ & $5.7 \pm 2.4$ & $6.9 \pm 2.3$ & $75.0 \pm 16.4$ \\
\hline Green/Purple DD HexHex & $4.5 \pm 1.2 \mathrm{a}$ & $9.5 \pm 2.8$ & $14.0 \pm 3.8$ & $100.0 \pm 0$ \\
\hline$F ; \mathrm{df} ; P$ & $6.64 ; 2,14 ; 0.009$ & $2.52 ; 2,14 ; 0.1$ & $1.66 ; 2,14 ; 0.2$ & \\
\hline
\end{tabular}

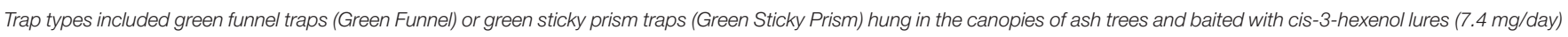

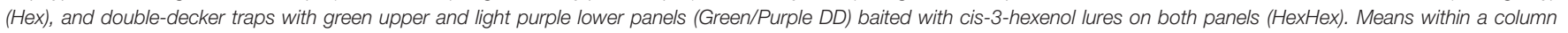
followed by different letters are significantly different, Tukey test, $P<0.05, N=8$.

and Phoebe oil lures. Previous studies have found that females were more attracted than males to purple prism traps hung in the canopy (Crook et al., 2009; Francese et al., 2010b, 2013a) and males were significantly more attracted than females to green prism traps baited with cis-3-hexenol (Grant et al., 2011; Crook et al., 2012; Ryall et al., 2012). The wavelength of purple is similar to the spectrum of tree bark, suggesting that females that typically spend time resting and ovipositing on the boles of ash trees (Rodriguez-Saona et al., 2007; Lelito et al., 2008) may be attempting to locate suitable host sites for oviposition. On the other hand, males typically hover around the canopies of ash trees and land and rest on leaves or females feeding in the canopy (Lelito et al., 2007, 2008; RodriguezSaona et al., 2007). Therefore, males may be more attracted to colors and volatiles associated with leaves in the canopy. Because more males were captured than females, overall more A. planipennis of both sexes were captured in light green than in dark purple sticky prism traps at the site with the lowest infestation level. Similarly, Francese et al. (2010b) found more A. planipennis were captured in green than in dark purple prism traps hung in the mid-canopy of ash trees. Our finding that significantly more $A$. planipennis were captured in light purple double-decker traps than in dark purple double-decker traps also support previous studies that found light Sabic purple was more attractive to A. planipennis than dark purple (Francese et al., 2010a, 2013a).

There was no significant difference in numbers of $A$. planipennis males or females captured on double-decker traps baited with cis-3-hexenol on both prisms compared to traps baited with cis-3-hexenol on the upper prism and Manuka oil on the lower prism among double-decker traps of the same color. cis-3-Hexenol is readily available from chemical suppliers as a synthetic green leaf alcohol and is relatively inexpensive and consistent in quality and supply. On the other hand, Manuka oil and Phoebe oil are natural steam distillates that can vary from batch to batch and supply may be inconsistent 
and limited. Although Phoebe oil has been shown to enhance attraction of $A$. planipennis to Manuka oil due to the presence of an additional antenally-active bark volatile (Crook et al., 2012), it was not available for inclusion in experiments 3 and 4 conducted in 2014 and 2015. Due to inconsistent and limited availability of natural oils, cis-3-hexenol would be preferred for operational use.

Differences in responses by males and females to different colors and lures under different conditions suggest that there is no single best trap color and lure. Double-decker traps incorporate both green and purple prisms at different heights which may attract beetles of both sexes. In all 3 experiments that included green/purple double-decker traps, they consistently had $100 \%$ detection rate.

The infestation level of trees in which sticky prism traps were hung was a significant covariate in the model. Not surprisingly, traps hung in trees ranked as heavily infested captured more beetles than traps hung in lightly infested trees. Similarly, Poland and Mccullough (2014) found a significant correlation between number of A. planipennis captured and infestation level of trees in which traps were hung and infestation level of trees in the vicinity of traps. In operational detection programs where A. planipennis is not known to be established there are unlikely to be any heavily infested trees.

Results for experiments conducted at sites with low infestation levels are most relevant to what is needed for operational detection surveys. Fox Memorial Park in experiment 3, and Legg Park in experiment 4 had very low A. planipennis infestations after peak outbreaks had declined. Most mature ash trees had died; however, there were abundant ash saplings at the sites. Although environmental and host conditions were different from a newly infested site, the very low $A$. planipennis populations would be similar to a new infestation. At these sites, the green/purple double decker traps and green funnel traps captured significantly more females than green sticky prism traps and had higher detection rates. Thus, they may be more effective for early detection programs.

Each of the trap designs has advantages and disadvantages. Sticky prism traps hung in the canopy are light, and easy to assemble and install. However, they are coated with glue, messy to handle, and cannot be reused. They are hung in the canopy and, therefore, require suitable host trees and extra equipment for hanging. Rope or string for hanging traps may become tangled, and the glue-coated prisms may become stuck in foliage making it difficult to raise and lower traps for checking. Double-decker traps are bulkier and although the T-posts and PVC pipes can be reused, the glue-coated prisms are messy and cannot be used again. In addition, they require twice as many prisms and lures as sticky prism traps hung in the canopy which increases per trap costs. They can be easily set up anywhere in the open near the edge of a stand and do not require suitable hosts, rope, or hanging equipment; however, they require custom assembly. Multiple funnel traps are not sticky and can be reused but are more expensive and must be hung in the canopy and, therefore, have some of the same challenges as sticky prism traps with hanging equipment and ropes that may become tangled. Collection cups require the use of a liquid or insecticidal killing agent and must be collected from every 3 to 4 weeks or captured insects may decay.

All trap contents are collected from the cups of multiple funnel traps and are later sorted and identified by experts in the laboratory; whereas field crews must recognize and collect insects suspected to be A. planipennis from the trapping glue on sticky prism traps. Although insects retrieved from sticky prism traps are verified by experts in the laboratory, it may be more likely that a positive trap capture on sticky prism traps might be missed by field crews than in multiple funnel traps.

It is generally assumed that an increased number of beetles captured corresponds to a higher probability of detection. However, in our experiments that was not necessarily true. In experiment 3, at the heavily infested site near Ithaca, MI, green funnel and green prism traps hung in the canopy captured significantly more $A$. planipennis on average than double-decker traps. However, variability in trap catches for traps hung in the canopy was much greater than for double-decker traps, possibly reflecting variable infestation levels of trees in which they were hung. Numbers of A. planipennis captured ranged from 8 to 180 (95\% difference) in dark green funnel traps and from 12 to 159 (92\% difference) in green prism traps, compared to a range from 15 to 31 (51\% difference) in green/purple double-decker traps. Overall, green/purple double-decker traps appeared to be most consistent and had 100\% detection rates at sites with very low to high infestations in all 3 experiments in which they were tested. Other studies have also found that double-decker traps consistently have higher detection rates than other traps (Marshall et al., 2010a,b; McCullough et al., 2011; Poland and Mccullough, 2014). Therefore, double-decker traps may be the most effective for detection and should be considered for areas where operational detection efforts are focused.

Many factors may interact and influence detection rate and number of $A$. planipennis captured: trap type, color, lure, trap placement within the canopy or in the open along the forest edge, sun exposure, ash host tree density, stand condition, infestation level of the tree in which the trap is hung, and A. planipennis population density. These and other factors should be considered in selection of trap type for detection surveys. Future research is needed to determine the effective trapping radius of different trap types and their accuracy in reflecting infestation level of a stand and attack densities in surrounding trees. Positive detection of A. planipennis in a trap currently does not provide detailed information about proximity of infested trees or site infestation levels, although number captured may provide an indication of general infestation level. Operational programs must also consider logistical issues including trap costs and longevity. While sticky prisms are initially less expensive than multiple funnel traps, they must be replaced each year, while multiple funnel traps may be reused for several decades. Future research is also needed to determine the cost benefit of different trap types and the optimal density of traps to deploy over a given survey area in relation to host density. 


\section{DATA AVAILABILITY STATEMENT}

The datasets generated for this study are available on request to the corresponding author.

\section{AUTHOR CONTRIBUTIONS}

TPo analyzed the data and wrote the first draft of the manuscript. TPo, TPe, and TC helped design the studies, conduct the field experiments, and edited the manuscript.

\section{REFERENCES}

Allison, J. D., Graham, E. E., Poland, T. M., and Strom, B. L. (2016). Dilution of fluon before trap surface treatment has no effect on longhorned beetle (Coleoptera: Cerambycidae) captures. J. Econ. Entomol. 109, 1215-1219. doi: $10.1093 /$ jee/tow081

Bartels, D., Williams, D., Ellenwood, J., and Sapio, F. (2008). “Accuracy assessment of remote sensing imagery for mapping hardwood trees and emerald ash borerstressed ash trees," in Emerald Ash Borer Research and Technology Development Meeting, 23-24 October 2007, Pittsburgh, PA: Department of Agriculture, Forest Service Publication FHTET-2008-07, eds V. Mastro, D. Lance, R. Reardon, and G. Parra (Morgantown, WV), 63-65.

Brockerhoff, E. G., Liebhold, A. M., Richardson, B., and Suckling, D. M. (2010). Eradication of invasive forest insects: concepts, methods, costs and benefits. $N$. Z. J. For. Sci. 40(Suppl.), S117-S135.

Burr, S. J., McCullough, D. G., and Poland, T. M. (2018). Density of emerald ash borer (Coleoptera: Buprestidae) adults and larvae at three stages of the invasion wave. Environ. Entomol. 47, 121-132. doi: 10.1093/ee/nvx200

CFIA (2019). Emerald Ash Borer - Agrilus Planipennis. Available Online at: https:// www.inspection.gc.ca/plants/plant-pests-invasive-species/insects/emeraldash-borer/eng/1337273882117/1337273975030

Crook, D., Khrimian, A., Francese, J. A., Fraser, I., Poland, T. M., Sawyer, A. J., et al. (2008). Development of a host-based semiochemical lure for trapping emerald ash borer, Agrilus planipennis (Coleoptera: Buprestidae). Environ. Entomol. 37, 356-365. doi: 10.1093/ee/37.2.356

Crook, D. J., Francese, J. A., Rietz, M. L., Lance, D. R., Hull-Sanders, H. M., Mastro, V. C., et al. (2014). Improving detection tools for emerald ash borer (Coleoptera: Buprestidae): comparison of multifunnel traps, prism traps, and lure types at varying population densities. J. Econ. Entomol. 107, 1496-1501. doi: 10.1603/EC14041

Crook, D. J., Francese, J. A., Zylstra, K. E., Fraser, I., Sawyer, A. J., Bartels, D. W., et al. (2009). Laboratory and field response of the emerald ash borer (Coleoptera: Buprestidae) to selected regions of the electromagnetic spectrum. J. Econ. Entomol. 102, 2160-2169. doi: 10.1603/029.102.0620

Crook, D. J., Khrimian, A., Cossé, A., Fraser, I., and Mastro, V. C. (2012). Influence of trap color and host volatiles on capture of the emerald ash borer (Coleoptera: Buprestidae). J. Econ. Entomol. 105, 429-437. doi: 10.1603/EC11204

Crook, D. J., and Mastro, V. C. (2010). Chemical ecology of the emerald ash borer Agrilus planipennis. J. Chem. Ecol. 36, 101-112. doi: 10.1007/s10886-0099738-x

de Groot, P., Grant, G. G., Poland, T. M., Scharbach, R., Buchan, L., Nott, R. W., et al. (2008). Electrophysiological response and attraction of emerald ash borer to green leaf volatiles (GLVs) emitted by host foliage. J. Chem. Ecol. 34, 1170-1179. doi: 10.1007/s10886-008-9514-3

Domingue, M. J., Lelito, J. P., Fraser, I., Mastro, V. C., Tumlinson, J. H., and Baker, T. C. (2013). Visual and chemical cues affecting the detection rate of the emerald ash borer in sticky traps. J. Appl. Entomol. 137, 77-87. doi: 10.1111/j.1439-0418.2012.01737.x

Domingue, M. J., Pulsifer, D. P., Lakhtakia, A., Berkebile, J., Steiner, K. C., Lelito, J. P., et al. (2015). Detecting emerald ash borers (Agrilus planipennis) using branch traps baited with 3D-printed beetle decoys. J. Pest Sci. 88, 267-279. doi: 10.1007/s10340-014-0598-y

\section{FUNDING}

This research was supported by federal funds from the USDA Forest Service.

\section{ACKNOWLEDGMENTS}

We thank Tom Baweja, Emily Loubert, and Elizabeth Graham for assistance with field experiments, John Stanovick for assistance with statistical analyses, and Robert Haack, Dan Miller, and two reviewers for comments on an earlier version of the manuscript.

Fierke, M. K., Whitmore, M. C., Foelker, C., Vandenberg, J. D., and Carlson, J. (2013). Delimitation and management of emerald ash borer (Coleoptera: Buprestidae): case study at an outlier infestation in southwestern New York State, United States of America. Can. Entomol. 145, 577-587. doi: 10.4039/tce.2013.39

Francese, J. A., Booth, E. G., Lopez, V. M., and Sorensen, B. (2019). Alternative survey methods for the emerald ash borer. FL. Entomol. 102, 243-245. doi: 10.1653/024.102.0142

Francese, J. A., Crook, D. J., Fraser, I., Lance, D. R., Sawyer, A. J., and Mastro, V. C. (2010a). Optimization of trap color for the emerald ash borer, Agrilus planipennis (Coleoptera: Buprestidae). J. Econ. Entomol. 103, 1235-1241. doi: 10.1603/EC10088

Francese, J. A., Fraser, I., Lance, D. R., and Mastro, V. C. (2011). Efficacy of multifunnel traps for capturing emerald ash borer (Coleoptera: Buprestidae): effect of color, glue, and other trap coatings. J. Econ. Entomol. 104, 901-908. doi: 10.1603/EC11038

Francese, J. A., Fraser, I., Rietz, M. L., Crook, D. J., Lance, D. R., and Mastro, V. C. (2010b). Relation of color, size, and canopy placement of prism traps in determining capture of emerald ash borer (Coleoptera: Buprestidae). Can. Entomol. 142, 596-600. doi: 10.4039/n10-041

Francese, J. A., Oliver, J. B., Fraser, I., Lance, D. R., Youssef, N., Sawyer, A. J., et al. (2008). Influence of trap placement and design on capture of the emerald ash borer (Coleoptera: Buprestidae). J. Econ. Entomol. 101, 1831-1837. doi: 10.1603/0022-0493-101.6.1831

Francese, J. A., Rietz, M. L., Crook, D. J., Fraser, I., Lance, D. R., and Mastro, V. C. (2013a). Improving detection tools for the emerald ash borer (Coleoptera: Buprestidae): comparison of prism and multifunnel traps at varying population densities. J. Econ. Entomol. 106, 2407-2414. doi: 10.1603/EC13013

Francese, J. A., Rietz, M. L., and Mastro, V. C. (2013b). Optimization of multifunnel traps for emerald ash borer (Coleoptera: Buprestidae) influence of size, trap coating and color. J. Econ. Entomol. 106, 2415-2423. doi: 10.1603/EC13014

Graham, E. E., and Poland, T. M. (2012). Efficacy of fluon conditioning for capturing cerambycid beetles in different trap designs and persistence on panel traps over time. J. Econ. Entomol. 105, 395-401. doi: 10.1603/EC11432

Grant, G. G., Poland, T. M., Ciaramitaro, T., Lyons, D. B., and Jones, G. C. (2011) Comparison of male and female emerald ash borer (Coleoptera: Buprestidae) responses to Phoebe oil and $(z)$-3-hexenol lures in light green prism traps. J. Econ. Entomol. 104, 173-179. doi: 10.1603/EC10197

Grant, G. G., Ryall, K. L., Lyons, D. B., and Abou-Zaid, M. M. (2010). Differential response of male and female emerald ash borers (Col., Buprestidae) to (Z)-3-hexenol and manuka oil. J. Appl. Entomol. 134, 1-8. doi: 10.1111/j.1439-0418.2009.01441.x

Haack, R. A., Baranchikov, Y., Bauer, L. S., and Poland, T. M. (2015). "Chapter 1: emerald ash borer biology and invasion history," in Biology and Control of Emerald Ash Borer, eds R. G. Van Driesche and R. C. Reardon (Morgantown, WV: USDA Forest Service, Forest Health Technology Enterprise Team, FHTET-2014-09), 1-13.

Haack, R. A., Jendek, E., Liu, H., Marchant, K. R., Petrice, T. R., Poland, T. M., et al. (2002). The emerald ash borer: a new exotic pest in North America. Newsl. Mich. Entomol. Soc. 47, 1-5. Available online at: https://www.nrs.fs.fed.us/pubs/ jrnl/2002/nc_2002_haack_001.pdf 
Herms, D. A., and McCullough, D. G. (2014). Emerald ash borer invasion of North America: history, biology, ecology, impacts, and management. Ann. Rev. Entomol. 59, 13-30. doi: 10.1146/annurev-ento-011613162051

Lelito, J. P., Böröczky, K., Jones, T. H., Fraser, I., Mastro, V. C., Tumlinson, J. H., et al. (2009). Behavioral evidence for a contact sex pheromone component of the emerald ash borer, Agrilus planipennis Fairmaire. J. Chem. Ecol. 35, 104-110. doi: $10.1007 / \mathrm{s} 10886-008-9583-3$

Lelito, J. P., Fraser, I., Mastro, V. C., Tumlinson, J. H., and Baker, T. C. (2008). Novel visual-cue-based sticky traps for monitoring of emerald ash borers, Agrilus planipennis (Col., Buprestidae). J. Appl. Entomol. 132, 668-674. doi: $10.1111 / j .1439-0418.2008 .01308 . x$

Lelito, J. P., Fraser, I., Mastro, V. C., Tumlinson, J. H., Böröczky, K., and Baker, T. C. (2007). Visually mediated 'paratrooper copulations' in the mating behavior of Agrilus planipennis (Coleoptera: Buprestidae), a highly destructive invasive pest of North American ash trees. J. Insect Behav. 20, 537-552. doi: 10.1007/s10905-007-9097-9

Liebhold, A. M., and Tobin, P. C. (2008). Population ecology of insect invasions and their management. Ann. Rev. Entomol. 53: 387-408. doi: 10.1146/annurev.ento.52.110405.091401

Lyons, J. E., Runge, M. C., Laskowski, H. P., and Kendall, W. L. (2008). Monitoring in the context of structured decision-making and adaptive management. J. Wildl. Manag. 72, 1683-1692. doi: 10.2193/2008-141

Marshall, J. M., Storer, A. J., Fraser, I., Beachy, J. A., and Mastro, V. C. (2009). Effectiveness of differing trap types for the detection of emerald ash borer (Coleoptera: Buprestidae). Environ. Entomol. 38, 1226-1234. doi: 10.1603/022.038.0433

Marshall, J. M., Storer, A. J., Fraser, I., and Mastro, V. C. (2010a). Efficacy of trap and lure types for detection of Agrilus planipennis (Col., Buprestidae) at low density. J. Appl. Entomol. 134, 296-302. doi: 10.1111/j.1439-0418.2009.01455.x

Marshall, J. M., Storer, A. J., Fraser, I., and Mastro, V. C. (2010b). "Multistate comparison of detection tools at low emerald ash borer densities," in Proceedings of the Emerald Ash Borer and Asian Longhorned Beetle Research and Technology Development Meeting, Pittsburgh, PA, 19-21 Oct. 2009, eds V. Mastro, D. Lance, R. Reardon, and G. Parra (Morgantown, WV: U.S. Department of Agriculture, Forest Service Publication), 124-125.

McCullough, D. G., Poland, T. M., Anulewicz, A. C., and Cappaert, D. (2009a). Emerald ash borer (Agrilus planipennis Fairmaire) (Coleoptera: Buprestidae) attraction to stressed or baited ash (Fraxinus spp.) trees. Environ. Entomol. 38, 1668-1679. doi: 10.1603/022.038.0620

McCullough, D. G., Poland, T. M., Cappaert, D., and Anulewicz, A. C. (2009b). Emerald ash borer (Agrilus planipennis) attraction to ash trees stressed by girdling, herbicide and wounding. Can. J. For. Res. 39, 1331-1345. doi: 10.1139/X09-057

McCullough, D. G., Siegert, N. W., Poland, T. M., Pierce, S. J., and Ahn, S. Z. (2011). Effects of trap type, placement and ash distribution on emerald ash borer captures in a low density site. Environ. Entomol. 40, 1239-1252. doi: 10.1603/EN11099

Musolin, D. L., Selikhovkin, A. V., Shabunin, D. A., Zviagintsev, V. B., and Baranchikov, Y. N. (2017). Between ash dieback and emerald ash borer: two Asian invaders in Russia and the future of ash in Europe. Baltic For. 23, 316-333.

Orlova-Bienkowskaja, M. J., Drogvalenko, A. N., Zabaluev, I. A., Sazhnev, A. S., Peregudova, H. Y., Mazurov, S. G., et al. (2019). Bad and good news for ash trees in Europe: alien pest Agrilus planipennis has spread to the Ukraine and the south of European Russia, but does not kill Fraxinus excelsior in the forests. BioRxiv. doi: 10.1101/689240

Orlova-Bienkowskaja, M. J. (2014). Ashes in Europe are in danger: the invasive range of Agrilus planipennis in European Russia is expanding. Biol. Inv. 16, 1345-1349. doi: 10.1007/s10530-013-0579-8

Petrice, T. R., Haack, R. A., and Poland, T. M. (2013). Attraction of Agrilus planipennis (Coleoptera: Buprestidae) and other buprestids to sticky traps of various colors and shapes. Gt. Lakes Entomol. 46, 13-30. Available online at: https://www.fs.fed.us/nrs/pubs/jrnl/2013/nrs_2013_petrice_001.pdf

Poland, T. M., and McCullough, D. G. (2006). Emerald ash borer: Invasion of the urban forest and the threat to North America's ash resource. J. For. 104, 118-124. doi: 10.1093/jof/104.3.118
Poland, T. M., and Mccullough, D. G. (2014). Comparison of trap types and colors for capturing emerald ash borer adults at different population densities. Environ. Entomol. 43, 157-170. doi: 10.1603/EN13137

Poland, T. M., McCullough, D. G., and Anulewicz, A. C. (2011). Evaluation of double-decker traps for emerald ash borer (Coleoptera: Buprestidae). J. Econ. Ent. 104, 517-531. doi: 10.1603/EC10254

Poland, T. M., and Rassati, D. (2018). Improved early detection of invasive forest insects - a review of current methods and research. J. Pest. Sci. 92, 37-49. doi: 10.1007/s10340-018-1004-y

Rassati, D., Faccoli, M., Petrucco Toffolo, E., Battisti, A., and Marini, L. (2015). Improving the early detection of alien wood-boring beetles in ports and surrounding forests. J. Appl. Ecol. 52, 50-58. doi: 10.1111/1365-2664.12347

Rodriguez-Saona, C., Poland, T. M., Miller, J. R., Stelinski, L. L., Grant, G. G., de Groot, P., et al. (2006). Behavioral and electrophysiological responses of the emerald ash borer, Agrilus planipennis, to induced volatiles of Manchurian ash, Fraxinus mandshurica. Chemoecol. 16, 75-86. doi: 10.1007/s00049-005-0329-1

Rodriguez-Saona, C. R., Miller, J. R., Poland, T. M., Kuhn, T. M., Otis, G. W., Turk, T., et al. (2007). Behaviours of adult emerald ash borer, Agrilus planipennis (Coleoptera: Buprestidae). Gt. Lakes Entomol. 40, 1-16. Available online at: https://scholar.valpo.edu/tgle/vol40/iss1/1

Ryall, K. (2015). Detection and sampling of emerald ash borer (Coleoptera: Buprestidae) infestations. Can. Entomol. 147, 290-299. doi: 10.4039/tce.2015.7

Ryall, K. L., Fidgen, J. G., Silk, P. J., and Scarr, T. A. (2013). Efficacy of the pheromone (3Z)-lactone and the host kairomone (3Z)-hexenol at detecting early infestation of the emerald ash borer, Agrilus planipennis. Entomol. Exp. Appl. 147, 126-131. doi: 10.1111/eea.12052

Ryall, K. L., Silk, P. J., Mayo, P., Crook, D., Khrimian, A., and Cossé, A. A. (2012). Attraction of Agrilus planipennis Fairmaire (Coleoptera: Buprestidae) to a volatile pheromone: effects of release rate, host volatile and trap placement. Environ. Entomol. 41, 648-656. doi: 10.1603/EN11312

SAS Institute (2012). OC User's Manual, Version 9.4. Cary, NC: SAS Institute.

Siegert, N. W., McCullough, D. G., Liebhold, A. M., and Telewski, F. W. (2014). Dendrochronological reconstruction of the epicentre and early spread of emerald ash borer in North America. Div. Distrib. 20, 847-858. doi: $10.1111 /$ ddi. 12212

Silk, P. J., Ryall, K., Lyons, D. B., Sweeney, J., and Wu, J. (2009). A contact sex pheromone component of the emerald ash borer, Agrilus planipennis Fairmaire (Coleoptera: Buprestidae). Naturwiss 96, 601-608. doi: 10.1007/s00114-009-0513-1

Silk, P. J., Ryall, K., Mayo, P., Lemay, M., Grant, G. G., and Crook, D. (2011). Evidence for a volatile pheromone in Agrilus planipennis Fairmaire (Coleoptera: Buprestidae) that increases attraction to a host foliar volatile. Environ. Entomol. 40, 904-916. doi: 10.1603/EN11029

Silk, P. J., Ryall, K., and Roscoe, L. (2019). Emerald ash borer, Agrilus planipennis (Coleoptera: Buprestidae), detection and monitoring in Canada. For. Int. J. For. Res. 10, 323-343. doi: 10.1093/forestry/cpz036

Tobin, P. C., Kean, J. M., Suckling, D. M., McCullough, D. G., Herms, D. A., and Stringer, L. D. (2014). Determinants of successful arthropod eradication programs. Biol. Invasions. 16, 401-414. doi: 10.1007/s10530-0130529-5

USDA-APHIS-PPQ (2018). 2018 Emerald Ash Borer Survey Guidelines. Available online at: http://www.aphis.usda.gov/plant_health/plant_pest_info/emerald_ ash_b/downloads/survey_guidelines.pdf

Yu, C. (1992). "Agrilus marcopoli Obenberger," in Forest Insects of China, 2nd Edn, ed G. Xiao (Beijing: China Forestery Publishing House), 400-401.

Conflict of Interest: The authors declare that the research was conducted in the absence of any commercial or financial relationships that could be construed as a potential conflict of interest.

Copyright $\odot 2019$ Poland, Petrice and Ciaramitaro. This is an open-access article distributed under the terms of the Creative Commons Attribution License (CC BY). The use, distribution or reproduction in other forums is permitted, provided the original author(s) and the copyright owner(s) are credited and that the original publication in this journal is cited, in accordance with accepted academic practice. No use, distribution or reproduction is permitted which does not comply with these terms. 